\title{
Simulating the dynamics of complex plasmas
}

\author{
M. Schwabe \\ Department of Chemical and Biomolecular Engineering, University of \\ California, Berkeley, CA 94720, USA \\ Max Planck Institute for extraterrestrial Physics, PO Box 1312, \\ Giessenbachstr., 85741 Garching, Germany \\ D. B. Graves \\ Department of Chemical and Biomolecular Engineering, University of \\ California, Berkeley, CA 94720, USA
}

\begin{abstract}
Complex plasmas are low temperature plasmas that contain micrometer-sized particles in addition to the neutral gas particles and the ions and electrons that make up the plasma. The microparticles interact strongly and display a wealth of collective effects. Here, we report on linked numerical simulations that reproduce many of the experimental results of complex plasmas. We model a capacitively coupled plasma with a fluid code written for the commercial package COMSOL. The output of this model is used to calculate forces on microparticles. The microparticles are modeled using the molecular dynamics package LAMMPS, which we extended to include the forces from the plasma. Using this method, we are able to reproduce void formation, the separation of particles of different sizes into layers, lane formation, vortex formation and other effects.
\end{abstract}

\section{Introduction}

In 1994, three research groups succeeded in forming crystals composed of small particles in a low temperature plasma, called "plasma crystals" [1]3. These systems ignited the interest of scientists world-wide, and the research on them has since increased dramatically. In general, charged microparticles embedded in a weakly ionized plasma are called "complex plasmas" in analogy to complex liquids - soft matter systems in the liquid form [4]. The term "complex plasma" sets these systems apart from the naturally occurring dusty plasmas, even though this distinction is not always made. 
Complex plasmas are of great interest as the microparticles can become the dominant species regarding energy and momentum transport [4], and they display a multitude of collective effects that occur in other systems as well. Complex plasmas can often serve as model for these other systems.

Numerical simulations of complex plasmas are useful to check the theoretical understanding, to make estimates of plasma parameters that are not easily accessible in experiments, and to predict parameters for future investigations. Three main approaches to simulations of complex plasmas exist, along with variations and hybrid models of these approches.

Firstly, the plasma is modeled as a fluid. If charging processes are investigated, applying a fixed potential at the surface of a stationary particle suffices [5]. In order to simulate larger dynamic systems, the microparticles are treated as an additional fluid in a simulation of the whole complex plasma [6-10]. Akdim and Goedheer [11] introduced tracer particles into such a model and were able to reproduce the vortices that often form in weightless complex plasmas.

These fluid models suffer from the problem that the time scale of the microparticle dynamics is much larger than that of the plasma, and the simulation typically switches periodically between advancing the plasma fluid and advancing the microparticle fluid, until an equilibrium is reached [12, 10]. The microparticles and the plasma are coupled via the Poisson equation and the forces acting on the microparticles.

The second approach to numerical simulations of complex plasmas is to use Particle-In-Cell (PIC) methods, often coupled with Monte Carlo (MC) simulations, to study the movement of ions and electrons in the vicinity of microparticles [13. This approach is very effective in investigating ion drag effects, wakes and the charging process of microparticles [14 33]. MC methods are also used to study ion transport in complex plasmas 34. The interaction between multiple microparticles is taken into account with a particle-particle-particle-mesh scheme. Microparticles are usually treated as stationary [13]. Some authors have also performed a molecular dynamics simulation of the plasma flow around stationary microparticles [35-37]. PIC methods can also be used to study the whole discharge with stationary dust [38, 39]. If the PIC is coupled to microparticle transport modules [40 42], dust distribution functions can be derived.

The third approach to modeling complex plasmas is to use moleculardynamics (MD)/Langevin dynamics simulations of the microparticles. The plasma is usually taken into account analytically via the microparticle interaction potential [e.g., 43, 18, 44 61]. A PIC analysis can determine the interaction potential more accurately and then serve as input for the MD simulation [e.g., 62, 63]. Other plasma effects can be taken into account analytically as well, for instance a fluctuating particle charge [64]. Schweigert et al. [65] use an MD simulation and include the effect of ion space charges by analytically including point charges in place of the ion space charges 
below the microparticles, as proposed in [16.

Hybrid approaches combine two or more of the techniques described above. For instance, Matyash et al. [27, 66] use a $\mathrm{P}^{3} \mathrm{M}$ model which combines PIC and MD approaches to study charging of microparticle strings. If nanoand microparticle growth is to be modeled, often, sectional models are used. These models can be coupled to fluid models of the plasma [see 67 and references therein]. Melands $\varnothing$ and Goree [68], Melands $\varnothing$ [69] use a hybrid approach in which they represent the plasma as a fluid and the microparticles as diffuse objects and study particle arrangement under the influence of the ion drag force. Yu et al. [70] combine a 2D sheath fluid model and a 3D microparticle transport model to study the formation of plasma crystals. Romé et al. [71] couple a fluid description of electrons to a kinetic description of dust.

Several authors in the group of M. Kushner have developed a series of linked computer models to investigate the trapping of microparticles in radio-frequency (rf) discharges [72-76]: A Monte-Carlo-fluid hybrid simulates the plasma, a PIC simulation determines microparticle charging and ion-microparticle momentum transfer cross section, and a plasma chemistry Monte Carlo simulation provides ion flux. This is coupled to a microparticle transport module that calculates microparticle trajectories. The authors were able to identify several regions in which microparticle trapping occurred. In the later models feedback to the plasma was included to investigate particle transport and Coulomb crystallization [75].

The model we present here follows a similar concept as [72 76]: We perform a hybrid fluid/analytical simulation of a radio-frequency plasma and couple this to a molecular dynamics simulation of the microparticles. In contrast to the work described above, which was mainly aimed at finding the trapping positions and studying plasma crystal formation, we reproduce dynamical collaborative effects in complex plasmas and compare with experimental results.

Both the capacitely-coupled plasma (CCP) model and the MD model are two-dimensional. The CCP model consists of a plasma sheath, which is solved analytically, and the plasma bulk, which is subject to the fluid simulation [77. The sheath width is fixed during the run of one simulation. This means that effects that depend on a changing sheath width, such as melting a plasma crystal by increasing the gas pressure [78, cannot be modelled accurately without extending the model. Also, we do not include the feedback from the microparticles to the plasma in this version of the model. This limits the applicability of the model to low microparticle densities where the Havnes parameter $\mathcal{H}$ [79] is less than unity:

$$
\mathcal{H}=n_{d} Z_{d} / n_{e} \ll 1 .
$$

Here, $n_{d}$ and $n_{e}$ designate the number densities of the microparticles and electrons and $Z_{d}$ the number of electrons on the microparticles. 


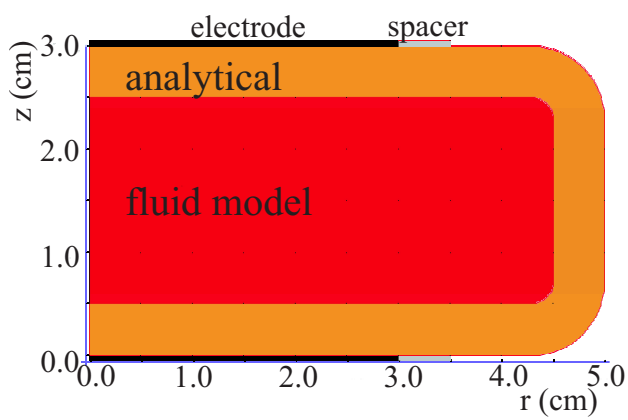

Figure 1: (color online) Modelled geometry, based on the PK-3 Plus chamber [82. Two parallel electrodes of radius $3 \mathrm{~cm}$ are separated by a distance of $3 \mathrm{~cm}$. The electrodes are surrounded by spacers of width $5 \mathrm{~mm}$. The nominal sheath width is set to $5 \mathrm{~mm}$. The sheaths are solved analytically, while the bulk plasma is treated with a fluid model. We only model the right half of the plasma chamber midplane and assume cylindrical symmetry.

\section{Plasma model}

Our capacitively-coupled plasma $(\mathrm{CCP})$ model is a modified version of the hybrid fluid-analytical simulation of inductive/capacitive discharges by Kawamura et al. [77, which itself is an extension of the plasma model by Hsu et al. [80] and the analytical sheath model by Lee et al. [81]. We base our model on the geometry of the PK-3 Plus chamber, which is a parallel-plate, capacitively coupled plasma chamber [82. Figure 1 shows a sketch of the geometry. The electrodes are separated by $3 \mathrm{~cm}$ and have a diameter of $6 \mathrm{~cm}$. We only model the right half of the experimental chamber, as it is approximately cylindrically symmetric. The electrodes are surrounded by a dielectric spacer and a grounded guard ring. In the physical experiment, the spacer is very small, but in the simulation, we use a spacer of width $5 \mathrm{~mm}$. The reason for this choice is that we have to integrate over the spacer to obtain some output values, such as the radio-frequency voltage, and the error in the integration becomes large when small spacers are used. A wider spacer does not significantly modify the results in the plasma bulk.

The bulk of the plasma is modeled as a quasi-neutral fluid. Assuming quasi-neutrality significantly speeds up the simulation compared to the alternative, solving the Poisson equation. The sheaths are solved analytically. We fix the nominal sheath width at $5 \mathrm{~mm}$ and take into account the physically varying sheath width by varying the dielectric constant. All inductive coupling that is present in [77] is removed from the model. We also do not model the gas flow or the temperature distribution of the neutrals and ions in order to make the model faster.

The model uses the gas pressure $p$, the ion temperature $T_{i}$ and the input current $I$ as input parameters. We always chose $T_{i}=300 \mathrm{~K}$ and model argon 


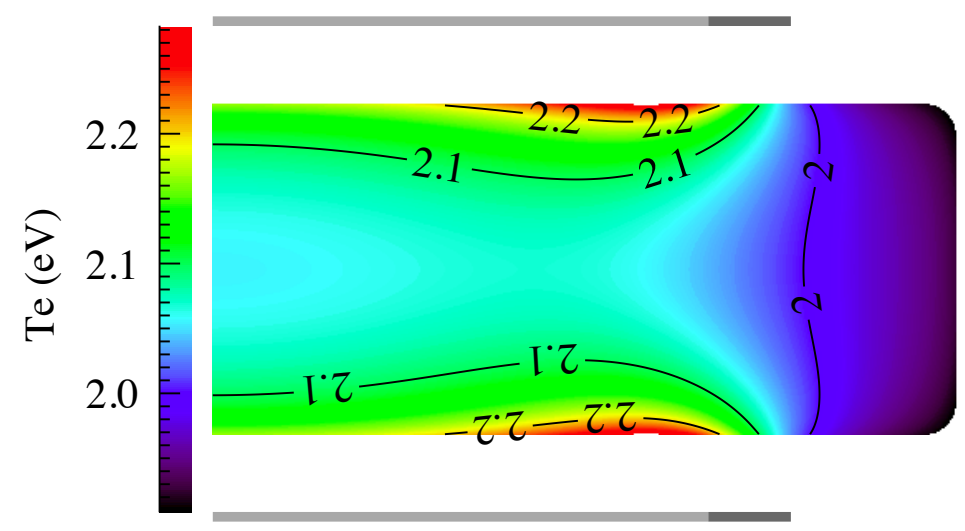

Figure 2: (color online) Electron temperature $T_{e}$ in the plasma bulk at a gas pressure of $20 \mathrm{~Pa}$ and an input current of $20 \mathrm{~mA}$. The gray lines in the top and bottom indicate the position of the electrodes and spacers.

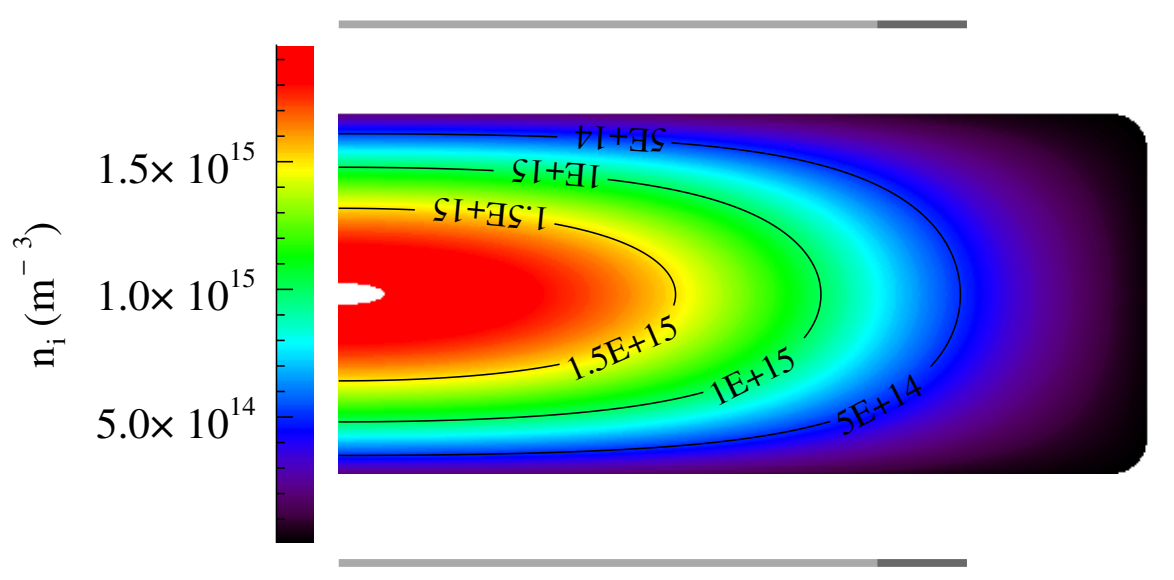

Figure 3: (color online) Distribution of ion density $n_{i}$ (and, via quasineutrality, electron density) in the plasma bulk at a pressure of $20 \mathrm{~Pa}$ and an input current of $20 \mathrm{~mA}$. The gray lines in the top and bottom indicate the position of the electrodes and spacers. 


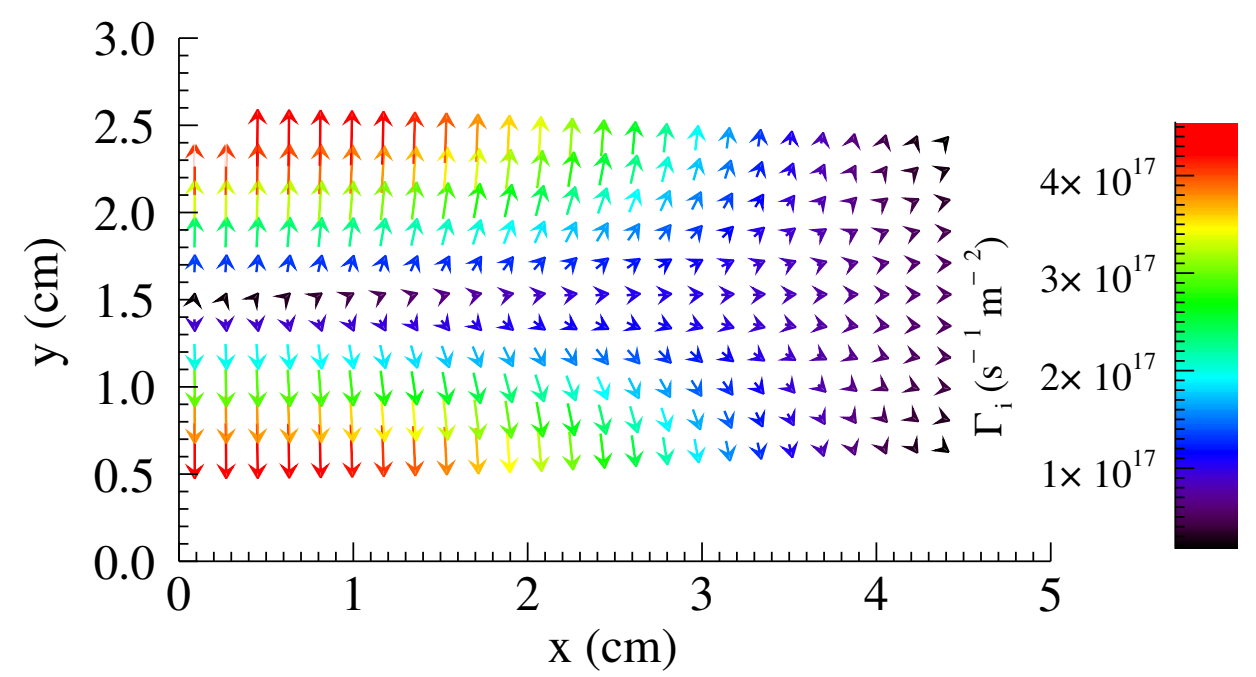

Figure 4: (color online) Ion flux $\Gamma_{i}$ in the plasma bulk as a function of position for a pressure $p=20 \mathrm{~Pa}$ and an input current $I=20 \mathrm{~mA}$.

as buffer gas. Without taking into account the influence of the microparticles on the plasma, quasi-neutrality causes the electron and ion densities to always be equal, $n_{i}=n_{e}$. The ion velocities are subject to the boundary condition that they reach Bohm velocity $u_{B}$ at the sheath edge, with $u_{B}=\sqrt{k_{B} T_{e} / m_{i}}$, where $T_{e}$ indicates the electron temperature, $k_{B}$ Boltzmann's constant, and $m_{i}$ the ion mass. The simulation results in the plasma densities, ion flux, electron temperature, ambipolar and rf electric fields, ionization rate, etc.

Even though we attempt to make the model as accurate as possible, taking into account various power deposition and energy loss mechanisms [77, we do not believe that this model is a quantitatively absolutely accurate description of the PK-3 Plus chamber. For instance, the currents used in the model are generally higher than those determined experimentally. Nevertheless, the model is qualitatively sufficient to reproduce many of the phenomena observed in the experiment, as we will discuss later. Before getting to the microparticle dynamics, we shall first discuss typical results of the plasma model.

Figure 2 shows the electron temperature distribution in the plasma bulk at a pressure of $20 \mathrm{~Pa}$ and an input current of $20 \mathrm{~mA}$. It can be seen that the electron temperature is highest near the electrodes, with a maximum close to the edge of the electrodes. The difference in electron temperature near the electrodes and in the center of the discharge is about $10 \%$. In contrast to the electron temperature, the electron and ion densities peak in the center of the discharge, as shown in Fig. 3. 


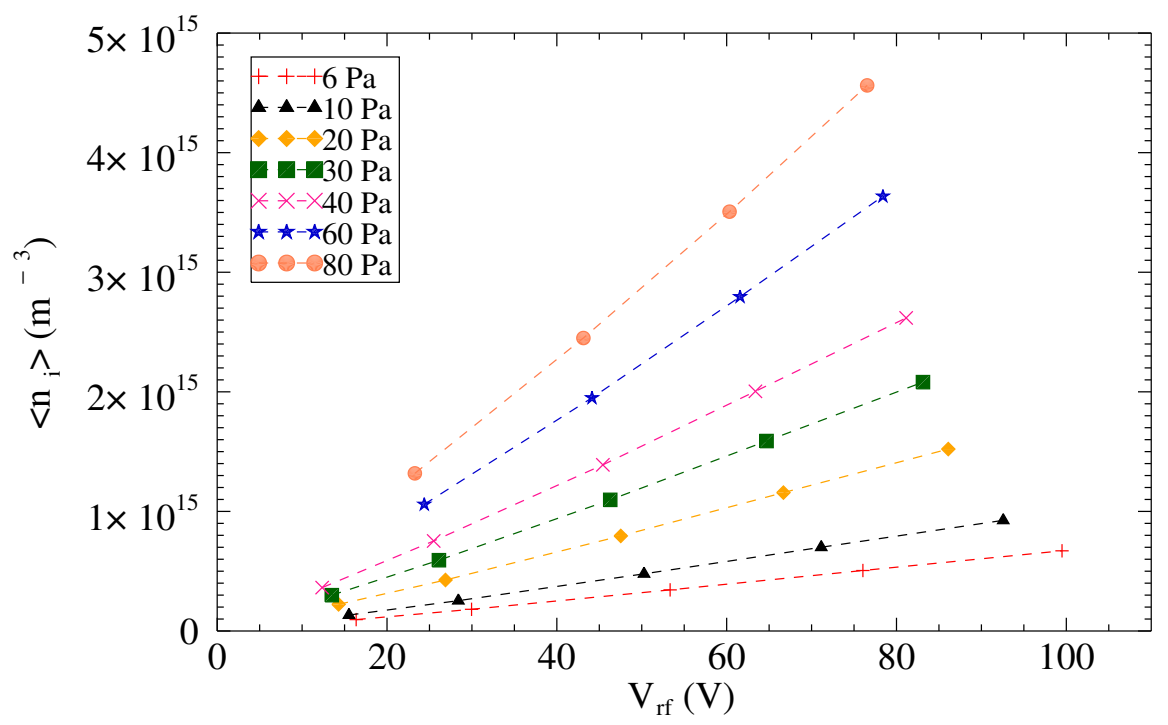

Figure 5: (color online) Mean ion density as a function of rf-voltage for different pressures. The lines are guides to the eye that connect series of constant pressure. The input currents used for each pressure were $I=5 \mathrm{~mA}$, $10 \mathrm{~mA}, 20 \mathrm{~mA}, 30 \mathrm{~mA}$, and $40 \mathrm{~mA}$. For the two highest pressures and lowest input current, the voltage falls below $10 \mathrm{~V}$, and the plasma model is no longer applicable. That is why we omit these data in the plot. The case shown in Fig. 2 and 3 corresponds to the voltage $V_{\text {rf }}=47.5 \mathrm{~V}$ and the pressure $p=20 \mathrm{~Pa}$. In general, the ion density increases with pressure and with voltage. 


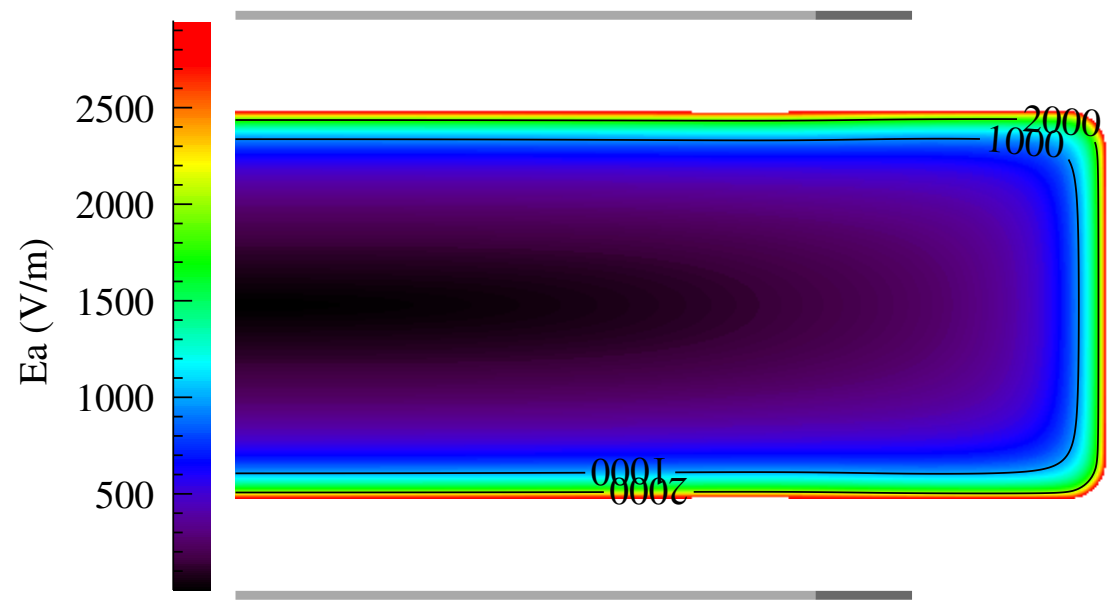

Figure 6: (color online) Magnitude of the ambipolar electric field $E a$ in the plasma bulk at a pressure of $20 \mathrm{~Pa}$ and an input current of $20 \mathrm{~mA}$. The gray lines in the top and bottom indicate the position of the electrodes and spacers.

The ions flow from the center of the plasma bulk outwards. They reach Bohm velocity at the sheath edge. The distribution of the ion flux is shown in Fig. 4

The ion and electron densities depend on the pressure and the input current/voltage, as can be seen in Fig. 5. They rise linearly with voltage. The mean density rises with pressure. Simultaneously, the ionization degree falls (not shown in the figure).

The densities of the plasma particles, the electron temperature and the ion flux have a profound impact on the dynamics of microparticles embedded in the plasma. The microparticles are confined in the bulk of the discharge by the ambipolar electric field that build up in and near the sheaths. Figure 6 shows the magnitude of the ambipolar electric field in the plasma bulk at a pressure of $20 \mathrm{~Pa}$ and an input current of $20 \mathrm{~mA}$. The field is strongest close to the sheaths and falls in the center of the chamber.

\section{Molecular dynamics model of the microparticles}

We use the freely available molecular dynamics (MD) code LAMMPS 83]. The microparticles are modelled as charged point particles that interact via a screened Coulomb potential $\Phi$

$$
\Phi=\frac{q_{1} q_{2}}{4 \pi \epsilon_{0} r} \exp \left(-r / \lambda_{D}\right),
$$


where $q_{1}$ and $q_{2}$ are the charges of the microparticles, $r$ is the distance between them, $\epsilon_{0}$ signifies the electric permittivity of the vacuum, and $\lambda_{D}$ is the Debye length. We use the Debye length calculated from the mean ion density resulting from the plasma simulation. In future refinements of the code, a local Debye length could be used, but this is not implemented yet. It is possible to use a charge of the microparticles that depends on the position - however, in the simulations presented in this paper, all microparticles carry the same charge. Typically, we estimate the average microparticle charge $q$ with Matsoukas and Russel [84]'s approximation

$$
q \approx C \frac{4 \pi \epsilon_{0} r_{d} k_{B} T_{e}}{e^{2}} \ln \frac{n_{i}}{n_{e}}\left(\frac{m_{e} T_{e}}{m_{i} T_{i}}\right)^{1 / 2} .
$$

As usual, $r_{d}$ is the radius of the microparticles, $k_{B}$ Boltzmann's constant, $e$ the electron charge, $n_{i}$ and $n_{e}$ the ion and electron densities, $m_{i}$ and $m_{e}$ their masses, and $T_{i}$ and $T_{e}$ their temperatures. For a typical argon plasma, the constant $\mathrm{C}$ is approximately $C \approx 0.73[84$.

Unless otherwise noted, we calculate the microparticle charge with Eq. 3 in the beginning of the simulation as a function of the mean plasma parameters. As alternative for specific simulations, we set the charge to a specific, user selected value.

The microparticles are embedded in a gas with a given temperature and interact with the gas. Firstly, there is friction with the gas when the microparticles are moving with a velocity $\boldsymbol{v}$ with respect to the gas. This results in the force

$$
\boldsymbol{F}_{\boldsymbol{E} \boldsymbol{p}}=-m_{d} \gamma \boldsymbol{v}
$$

where $m_{d}$ is the mass of the microparticles, and $\gamma$ is the coupling constant. We use Epstein [85] 's well-known formula to calculate $\gamma$, using the coefficient $\delta=1.48$ as determined experimentally for complex plasmas [86]:

$$
\gamma=\delta \frac{n_{n} m_{n} u_{n}}{\rho_{d} r_{d}} .
$$

Here, $n_{n}, m_{n}$, and $u_{n}$ signify the number density, mass, and thermal velocity of the neutral particles, respectively. The microparticle mass density and radius are given by $\rho_{d}$ and $r_{d}$.

Secondly, the gas not only reduces the microparticle velocity, it also transfers energy to the microparticles via heating. This is modeled by random kicks to the microparticles that bring the microparticles to the same temperature as the background gas. The resulting force $\boldsymbol{F}_{\boldsymbol{r}}$ is proportional to

$$
F_{r} \propto \sqrt{\frac{k_{B} T_{n} m_{d} \gamma}{d t}}
$$

with $d t$ being the time step of the simulation and $T_{n}$ the gas temperature. The force is applied on a per-particle basis. The direction and magnitude is randomized using uniform random numbers [87]. 
We have modified the LAMMPS source code to include the influence of the plasma. For this purpose, the ion and electron densities, electron temperature, ion velocities and ambipolar electric fields are written to an ascii file by Matlab using the information from COMSOL. Our $\mathrm{C}++$ routine reads this file and interpolates the information to the positions of the microparticles.

The microparticles with charge $q_{d}$ react directly to the ambipolar electric field $\boldsymbol{E}_{\boldsymbol{a}}$ that is output from the plasma simulation. This results in the electric force

$$
\boldsymbol{F}_{\boldsymbol{e}}=q_{d} \boldsymbol{E}_{\boldsymbol{a}}
$$

We calculate the ion drag force $\boldsymbol{F}_{\boldsymbol{i} \boldsymbol{d}}$ using the same approach as Goedheer et al. [88], namely by incorporating results from previous studies by Khrapak et al. [89, Ivlev et al. [90], and Hutchinson [24]:

$$
\begin{aligned}
\boldsymbol{F}_{\boldsymbol{i d}}= & n_{i} m_{i} u_{i} \boldsymbol{v}_{\boldsymbol{i}}\left(\sigma_{c}\left(u_{i}\right)+\right. \\
& \left.\pi \rho_{0}\left(u_{i}\right)^{2}\left[\Lambda\left(u_{i}\right)+\mathcal{K}\left(\frac{\lambda_{D}\left(u_{i}\right)}{l_{\mathrm{mfp}}}\right)\right]\right) .
\end{aligned}
$$

Here, $n_{i}$ is the ion number density, $m_{i}$ the ion mass, $u_{i}$ the mean ion velocity, $\boldsymbol{v}_{\boldsymbol{i}}$ the ion velocity, $\sigma_{c}$ the cross section for ion capture, $\rho_{0}$ the Coulomb radius, $\Lambda$ the Coulomb logarithm and $\mathcal{K}$ a collisional function to take into account loss of angular momentum in collisions of ions and neutrals [90]. The Debye length $\lambda_{D}$ is the linearized Debye length based on the ordinary electron Debye length and the ion Debye length derived from the total ion energy. The ion mean free path is denoted by $l_{\mathrm{mfp}}$. The ion capture cross section $\sigma_{c}$ is a function of the radius of the microparticles $r_{d}$ and the Coulomb radius $\rho_{0}=Z_{d} e^{2} / 2 \pi \epsilon_{0} m_{i} u_{i}^{2}$, where $Z_{d}$ denotes the number of electron charges on the microparticles, $\epsilon_{0}$ the vacuum permittivity, and $e$ the electron charge:

$$
\sigma_{c}\left(u_{i}\right)=\pi r_{d}^{2}\left(1+\frac{\rho_{0}\left(u_{i}\right)}{r_{d}}\right) .
$$

The Coulomb logarithm $\Lambda$ is used in the calculation of the cross-section for scattering of ions around the microparticle. Following Khrapak et al. [89], we include ions scattered at a distance larger than the Debye length, and use

$$
\Lambda\left(u_{i}\right)=\ln \left[\frac{\rho_{0}\left(\tilde{u}_{i}\right)+\lambda_{D}\left(\tilde{u}_{i}\right)}{\rho_{0}\left(\tilde{u}_{i}\right)+r_{d}}\right] .
$$

In the calculation of the Coulomb logarithm, the expression of the mean ion velocity $u_{i}$ is modified from its ordinary value, $u_{i}^{2}=8 k_{B} T_{g a s} / \pi m_{i}+\boldsymbol{v}_{\boldsymbol{i}}{ }^{2}$, to fit a PIC simulation also at higher ion flow speeds. We use the correction 
factor given in [24], rather than the simpler one from [91] used in [88]:

$$
\begin{aligned}
& \tilde{u}_{i}^{2}=\frac{8 k_{B} T_{\text {gas }}}{\pi m_{i}}+\boldsymbol{v}_{\boldsymbol{i}}^{2}(1+ \\
& \left.\left[\frac{\left|\boldsymbol{v}_{\boldsymbol{i}}\right| / u_{B}}{0.6+0.05 \ln \left(m_{a}\right)+\left(\lambda_{D e} / 5 r_{d}\right)\left(\sqrt{T_{i} / T_{e}}-0.1\right)}\right]^{3}\right) .
\end{aligned}
$$

Here, $m_{a}$ denotes the atomic mass of the ions, $m_{a}=40$ for Argon. We also assume singly charged ions. The Bohm velocity $u_{B}$ is given by $u_{B}=$ $\sqrt{k_{B} T_{e} / m_{i}}$.

The collisional function $\mathcal{K}$ is given by 90

$$
\begin{aligned}
\mathcal{K}(x)= & x \arctan (x)+\left(\sqrt{\frac{\pi}{2}}-1\right) \frac{x^{2}}{1+x^{2}}- \\
& \sqrt{\frac{\pi}{2}} \ln \left(1+x^{2}\right) .
\end{aligned}
$$

We do not model the distribution of neutral temperature in the chamber and thus do not take into account the thermophoretic force. Even though we do have a slight electron temperature gradient (see Fig. 2), so far we do not take into account the force due to the electron temperature gradient 92.

Finally, after all forces are determined, the positions and velocities of the microparticles are updated with the Velocity Verlet algorithm [87]. We usually use time steps of $0.1 \mathrm{~ms}$ or $1 \mathrm{~ms}$, depending on the velocity of the microparticles. We use a cutoff of the potential of $2 \mathrm{~mm}$ for very dense complex plasmas up to $1 \mathrm{~cm}$ for very dilute complex plasmas.

\section{Collective particle effects}

Complex plasmas display a wealth of collective effects. In the following, we shall discuss some effects that our model is able to reproduce. Note that our model is two-dimensional (2D), whereas experiments with complex plasmas under microgravity are always three-dimensional (3D). This leads to a limited comparability, for instance, crystallization occurs for lower coupling parameters in 2D than 3D [93. Correspondingly, the particles in our simulation are often in crystalline state, whereas they are still fluid in 3D experiments.

All examples shown model an argon plasma containing spherical melamineformaldehyde particles with a mass density of $\rho_{d}=1510 \mathrm{~kg} / \mathrm{m}^{3}$.

\subsection{Confinement and void formation}

In experiments, microparticles are often injected into the plasma chamber with a dispenser that is mounted in the electrode [94] or near it [82, 95], or 

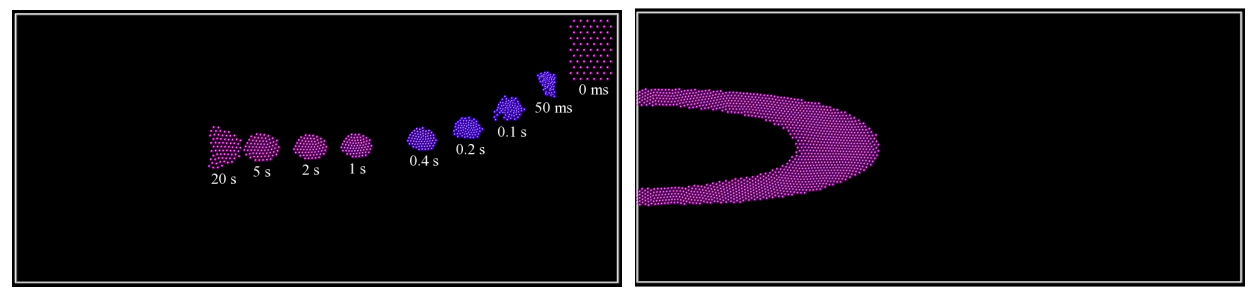

Figure 7: (color online) Formation process of a particle cloud with (left) a superposition of nine frames during the injection process and (right) the equilibrium particle cloud after 20 injections and a time span of 3.5 min after the last injection. The gas pressure is $20 \mathrm{~Pa}$, the input current $25 \mathrm{~mA}$. The gray box in the images indicates the dimensions of the simulated plasma bulk - one half of the vertical midplane of the plasma chamber without sheaths, with dimensions $4.5 \times 2 \mathrm{~cm}^{2}$. The center line of the plasma chamber corresponds to the left gray line at the edge of the field of view. The color of the microparticles indicates the vertical velocity $v_{z}$, ranging from blue/light gray for $-2 \mathrm{~mm} / \mathrm{s}$ to red (not reached in the figure) for $2 \mathrm{~mm} / \mathrm{s}$. Purple/dark gray color indicates no movement in $z$ direction, as for the time $t=0 \mathrm{~ms}$ in the left panel.

from the side [96]. The microparticles are charged very quickly compared to the time they need to fall from the dispenser to the lower electrode. Once charged, they are subject to the electric confinement and ion drag forces. Close to the sides of the chamber, the confinement force dominates and pushes the microparticles into the chamber, where they form a cloud. Near the center, the ion drag force dominates over the confinement force and pushes the microparticles towards the edges of the plasma. This leads to the formation of the particle-free central void.

At some distance from the chamber center, the confinement, interparticle and ion drag forces balance, and the particle cloud forms. This mechanism has been modeled before, see for instance [88, 10.

In order to mimic the injection process in the simulation, we place a small number of particles with no initial velocity near the upper right edge of the plasma bulk, close to the sheath. This is shown in the left panel of Fig. 7 with the label " $0 \mathrm{~ms}$ ". Then we let the simulation run its course. The particles are immediately accelerated towards the center of the chamber, as can be seen in the subsequent time steps in Fig. 7. The particle cloud quickly takes a round shape. This particle droplet then moves towards the plasma chamber. It is slowed progressively down by Epstein and ion drag while it approaches the center, and at some point the particles closer to the top of the droplet start moving upwards. This is visible in Fig. 7 as a change in the color of the microparticles. Once the particles approach the chamber center, the void clearly indents the particle droplet, and the microparticles 
start flowing around the void edge to the top and bottom. The beginning of this process can be seen in the last time step in Fig. 7.

We note that the particles move more slowly in the simulation than in experiments - for similar settings, the particles spread around the void within a few seconds in experiments. In the simulation, the particles have just reached the edge of the void after $20 \mathrm{~s}$. The reason for this is probably that in experiments, the particles are accelerated more strongly during the injection process: They transverse the sheath region on their way to the chamber center, where the electric field is much stronger than in the presheath region. In the simulation, we treat the sheath analytically, and thus do not allow particles to enter the sheath. This leads to significantly smaller accelerations of the microparticles.

Once the microparticles have reached the void, they spread around it. The right panel of Fig. 7 shows the equilibrium cloud after 20 particles injections such as shown in the left panel of Fig. 7, and after the cloud has evolved for 3 minute. More particles are arranged to the side than above and below the void, as observed in experiments. There is one layer of particles at the left edge of the simulation domain - the center of the plasma chamber. Here, we employ reflective boundary conditions, and there is no confinement field that would push the particles away from the edge as at all other edges. The pressure from the surrounding particles and the boundary lead to crystallization at this domain boundary. In experiments, it is often observed that particles crystallize above or below the void, but not necessarily in vertical strings as is induced by the boundary here.

In the simulations, the void seems slightly more oval than in the experiments. This might be due to an imperfect choice of the sheath width. We will explore this topic in more detail in the future. In the future, we will also study how a three-dimensional simulation changes the observed injection process. Experiments, e.g. on lane formation [97], have shown that particles generally move within the plane in which they were injected, even before they start to interact with particles that are already inside the chamber, so we do not expect a large deviation from the behavior we observe here.

In general, the distribution of particles around the void is stable, as long as the input parameters are not changed. The positions of individual particles are not necessarily stable, as we shall see in the next following.

\subsection{Vortices}

Vortices in complex plasmas are common [98,100, 94, 101]. In the PK-3 Plus setup, they occur at the edges of the cloud and are especially strong at lower pressures [99]. Figure 8 shows overlays of particle streamlines and average velocities recorded at a pressure of $10 \mathrm{~Pa}$. The particles move along the outer edges of the cloud towards the cloud center. When they reach the region 
above or below the void, they move into the cloud and back outwards. This direction of the vortices is not always the same, under some experimental conditions, they can reverse direction (e.g., particles move towards the void in the center of the cloud and back outwards along the cloud edge.)

The mean measured velocity of the particles in the vortices shown in Fig. 8 is of the order of $1 \mathrm{~mm} / \mathrm{s}$.

Akdim and Goedheer [11] modeled vortices in complex plasmas using tracer particles in a fluid simulation. They explain the formation of the vortices as follows: There is one equilibrium position in the midplane of the chamber where the confinement force equals the ion drag force. A single particle will find this position and stay there. As soon as other particles are present, they displace the particle from the equilibrium position into a region where the total force is not conservative. It is there that vortices form. This explanation is confirmed by an MD simulation [49]. It has been speculated that charge fluctuations play a role in vortex formation [47]however, Akdim and Goedheer [11] do not find charge differences necessary for vortex formation.

We confirm this result. Charge fluctuations are not included in our model, and the vortices form nevertheless. In contrast to [11, in our model, we do not only find one stable position in the chamber midplane, but a stable circle around the void. If the neutral gas pressure is low enough, vortices appear when two or more layers of microparticles are present and their positions are only slightly removed from the equilibrium line.

Figure 9 shows particle streamlines and velocities in the simulation. Vortices are present in which the particles move towards the center of the cloud along the cloud edges and outwards in the vertical cloud center. The velocity of the particles depends on the particle charge. In the figure, we chose a charge of $1 / 2$ of that given by Eq. (3), which results in velocities of up to $2.6 \mathrm{~mm} / \mathrm{s}$.

We also have so far not encountered vortices that rotated in the reversed direction in the simulation. Another difference to the experimental observations is that the vortices in our simulations envelop a larger region - they reach almost to the horizontal center, leaving only a few particles that are in crystallized state and don't rotate above and below the void. In the example shown in Fig. 8, the void is also surrounded by a circle of non-rotating particles - probably smaller contaminants. In the experiments, there are typically more particles in the regions above and below the void that do not participate in the rotation. In experiments, more complicated structures with several vortices along the horizontal axis and at the outer edges also occur 94.

We shall leave the topic of vortices for now and instead complicate the situation by introducing a second particle type. 


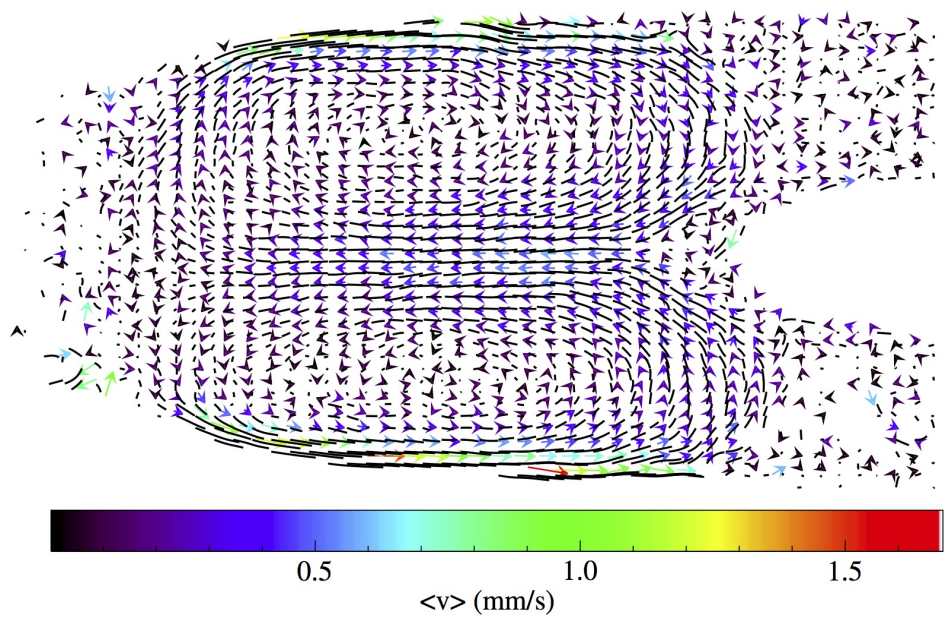

Figure 8: (color online) Vortices composed of particles of diameter $3.4 \mu \mathrm{m}$ in the PK-3 Plus setup under microgravity conditions in argon at a pressure of $10 \mathrm{~Pa}$. The black lines show the streamlines of the particle movement. The vector field of the mean particle velocities is overlaid. The color and length of the vectors indicates the total velocity, their orientation the direction of movement. Dimensions of the particle cloud: $33 \mathrm{~mm} \times 16 \mathrm{~mm}$. Data courtesy of the PK-3 Plus team 82].

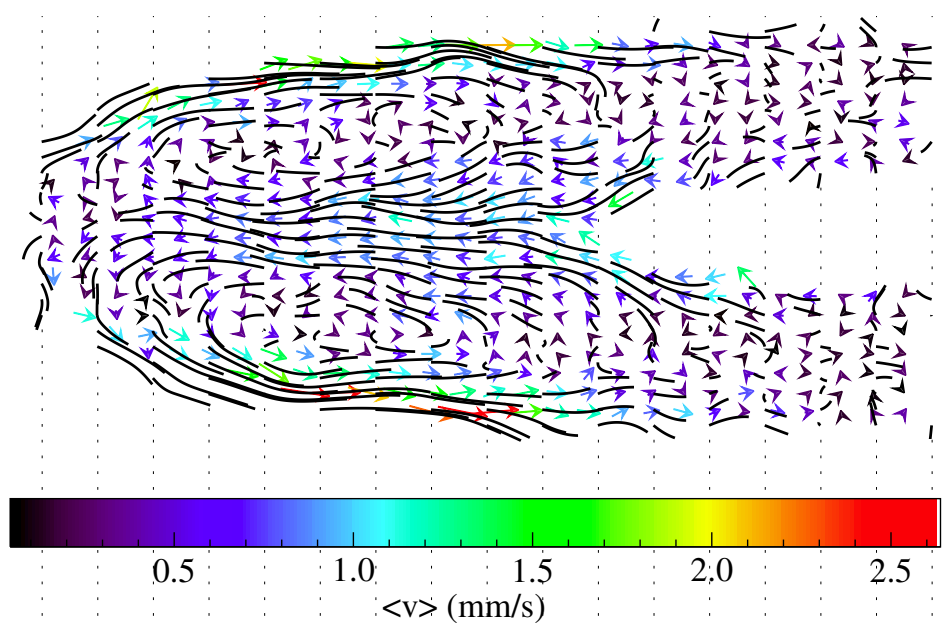

Figure 9: (color online) Simulated vortex for a pressure of $10 \mathrm{~Pa}, 3.4 \mu \mathrm{m}$ diameter particles, and an input current of $16 \mathrm{~mA}$. The particle charge was set to $1 / 2$ of that obtained with OML theory. Dimensions of the particle cloud: $23 \mathrm{~mm} \times 10 \mathrm{~mm}$. The streamlines of the particle movement are shown in black, analogous to Fig. 8. Superimposed over this are the mean particle velocities. The length and the color of the vectors indicates the total velocity, their orientation the direction of movement. The image was mirrored to the left side of the void to ease comparison with Fig. 8. 


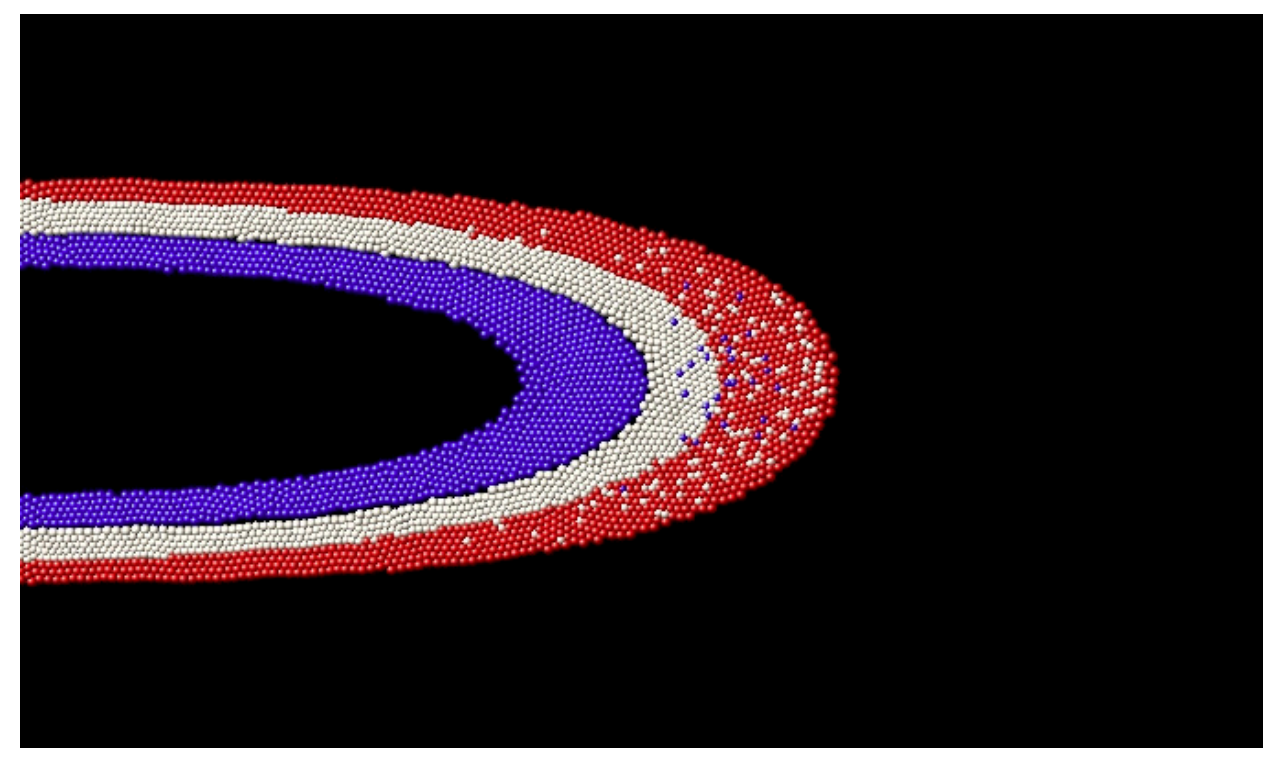

Figure 10: (color online) Particle cloud made up of three different particle sizes: $6.8 \mu \mathrm{m}$ (red/light gray), $3.4 \mu \mathrm{m}$ (white), and $1.55 \mu \mathrm{m}$ (blue/dark gray) diameter at a pressure of $20 \mathrm{~Pa}$ and a current of $30 \mathrm{~mA}$. Field of view: $33.9 \mathrm{~mm} \times 20.0 \mathrm{~mm}$. The particles were injected in five sets, each composed of a bunch of each particle size. Within each set, the largest particles were injected first. After the injections, the cloud was left alone to evolve for $3.5 \mathrm{~min}$, during which it sorted by particle size. Even after this time, the sorting is not complete - there are still some smaller particles mixed into the larger ones further out.

\subsection{Separation by particle size}

When particles of different sizes are injected into a plasma, they arrange around the void in layers - the smaller particles closer to the void than the larger ones. The reason for this is that the confinement force and the ion drag force have a different dependence on the particle radius, so that the equilibrium positions of the different particle sizes vary.

This has previously been modeled by Liu et al. [52], Liu and Chew [53] via an MD simulation of microparticles in a quadratic potential. The particles are subject to an outwards pointing drag force. The authors vary the strength of the drag force and produce various configurations of microparticles, with the different sizes mixed, the larger particles on the outside or the inside of the smaller ones.

In our case, we use the expressions for the confinement force and the ion drag force given in Sec. 3 and inject particles by placing them at the edge of the simulation box, as described in Sec. 4.1. The particles automatically arrange in layers as observed in experiments. Figure 10 shows a particle 


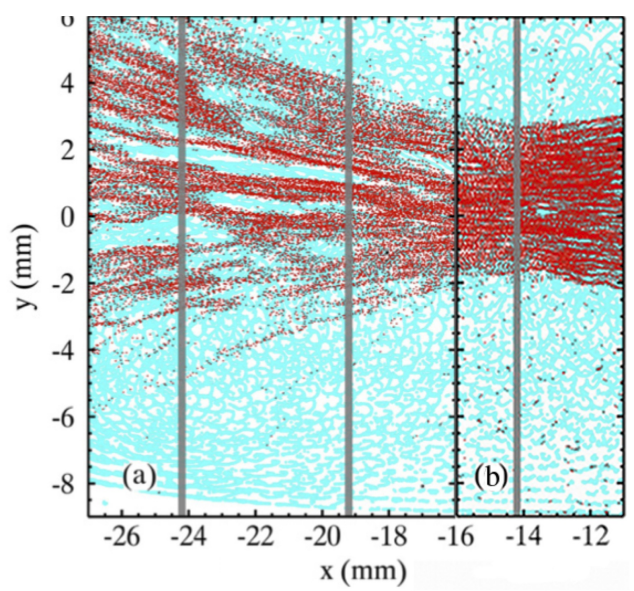

Figure 11: (color online) Lane formation in the PK-3 Plus laboratory on board the International Space Station. Microparticles of $3.4 \mu \mathrm{m}$ diameter, shown in red/dark gray, move through a cloud of $6.8 \mu \mathrm{m}$ diameter particles. The buffer gas is argon at a pressure of $30 \mathrm{~Pa}$. The crossover from lane formation (panel a) to phase separation (panel b) is visible. Courtesy of Du et al. 102]. (CIOP Publishing Ltd and Deutsche Physikalische Gesellschaft. Published under a CC BY-NC-SA licence.

cloud composed of microparticles of three sizes that autonomously arranged in layers. We did not observe homogeneous mixing of the particle sizes or a reverse of the positions as in Liu et al. [52, in accordance with the experiments. We did, however, observe another effect that is common in complex plasmas, which we shall discuss next.

\subsection{Lane formation}

Lanes form in complex plasmas when microparticles are injected into a cloud a larger microparticles. As we have just discussed, the particles arrange by size - the smaller particles move to the central region through the cloud of larger particles. Often, they do so by forming lanes [97, 57, 102]. During this process, the particles go through three stages [102]. Firstly, the ambipolar electric field pushes them towards the cloud of background particles. Secondly, once they enter the cloud, they form lanes. The lanes are made up of both small and large particles. Next, a crossover to the third stage, phase separation, occurs: The small particles move closer together, forming a drop inside the cloud of large particles. During the crossover, some large particles are left over inside the droplet of small particles and form lanes inside this droplet. Finally, all large particles are expelled, and a droplet of small particles moves towards the center of cloud.

Figure 11 by Du et al. [102] demonstrate this crossover. Small particles 


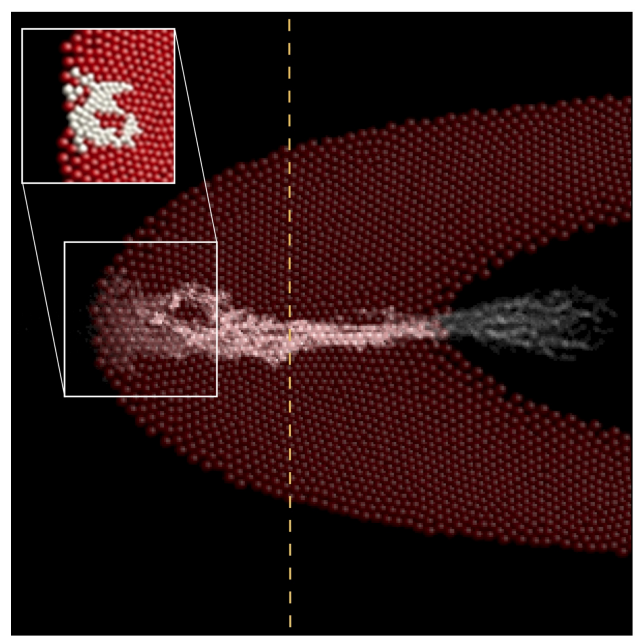

Figure 12: (color online) A batch of microparticles of $3.4 \mu \mathrm{m}$ diameter (white) in a cloud of particles with $6.8 \mu \mathrm{m}$ diameter, moving towards the center of the chamber. In the background, the positions of the small particles are overlaid on the background cloud during their voyage towards the center. The total field of view is $15 \mathrm{~mm} \times 15 \mathrm{~mm}$. The inset (field of view: $3.8 \mathrm{~mm} \times 3.8 \mathrm{~mm}$ ) shows one frame when the microparticles start penetrating into the background cloud, starting to form lanes. The gas pressure is $10 \mathrm{~Pa}$, input current $12 \mathrm{~mA}$. In contrast to the other examples shown in this paper, here, the sheath width is set to $3 \mathrm{~mm}$, and the temperature that the microparticles are subjected to from the background gas is $600 \mathrm{~K}$. The vertical dashed line marks the approximate position where lane formation turns into phase separation.

of $3.4 \mu \mathrm{m}$ diameter are injected into a cloud of $6.8 \mu \mathrm{m}$ diameter particles. First, they form lanes, then the small particles compress into a droplet, from which the larger particles are expelled.

Figure 12 shows simulated lane formation in argon at a pressure of $10 \mathrm{~Pa}$ and an input current of $12 \mathrm{~mA}$. In contrast to the other simulations in this paper, this one was run with a sheath width of $3 \mathrm{~mm}$, and the temperature that the gas transfers to the microparticles was $600 \mathrm{~K}$. The large particles, shown in red/dark gray, have a diameter of $6.8 \mu \mathrm{m}$, the small particles, shown in white, have a diameter of $3.4 \mu \mathrm{m}$, as in the experiments of $\mathrm{Du}$ et al. [102]. We use the microparticle charges determined in [102], namely $-4500 \mathrm{e}$ and $-1900 \mathrm{e}$.

As in [102, we observe all three stages - driven movement towards the cloud, lane formation, and crossover to phase separation -during the course of the simulation. In Fig. 12, the approximate point where the cross-over from lane formation to phase separation occurs is marked with a vertical dashed line. The driven movement of the small particles towards the cloud 


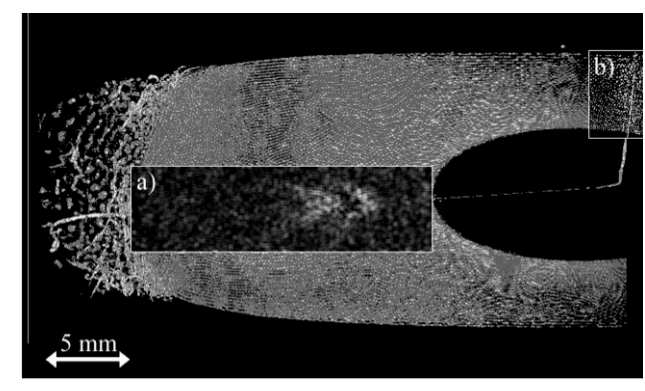

Figure 13: Mach cone excited by a projectile moving through a cloud of $2.55 \mu \mathrm{m}$ diameter particles at a gas pressure of $10 \mathrm{~Pa}$. The experiment was performed under microgravity in the PK-3 Plus laboratory on board the International Space Station. Inset a) shows the difference between two successive frames, making visible the Mach cone as difference in the particle densities. Inset b) is a superposition of 64 frames that shows the continuation of the trajectory upwards. Courtesy of Schwabe et al. [103].

is not visible in this figure. This stage is identical to the injection process shown in Fig. 7.

A difference between the simulation and the experiment is that the particles move significantly more slowly to the center of the cloud, and the crossover to phase separation occurs much faster. This is probably due to the lower injection speed (see paragraph on confinement and void formation) and to the fact that the cloud of bigger particles is partly crystallized in the simulation. Also, there are vortices present in the simulation, which suppress the lanes formed further away from the axis of symmetry. Another difference is that the lane formation in the beginning is less pronounced in the simulation, with fewer lanes of the larger particles, probably for the same reasons as the lower speed. The two-dimensional nature of our simulation also means that the microparticles do not interact with any out-of-plane particles, as they do in experiments. We will investigate this in more detail in future, three-dimensional simulations.

Next, we shall see what happens when a single fast, large particle penetrates the cloud instead of many small ones.

\subsection{Mach cones}

Mach cones form when a projectile moves through a fluid faster than the speed of sound. This is easy to achieve in complex plasmas where the speed of sound is typically of the order of a few $\mathrm{cm} / \mathrm{s}$. In the PK-3 Plus laboratory on board the International Space Station, sometimes bigger particles from the edge of the plasma cloud accelerate and move through the cloud [104, 103. The reason for the sudden acceleration is not completely understood 


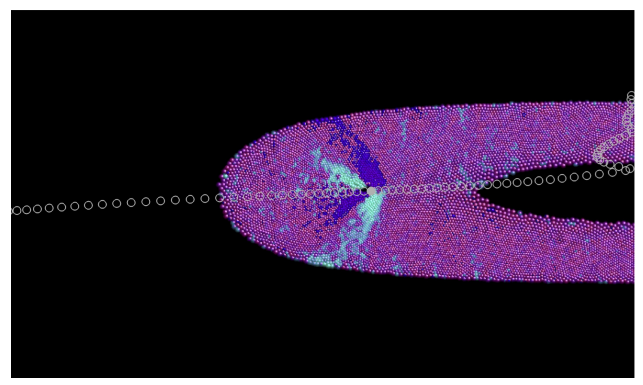

Figure 14: (color online) Simulated Mach cone in a cloud of $2.55 \mu \mathrm{m}$ diameter particles at a gas pressure of $10 \mathrm{~Pa}$. Field of view: $33.9 \mathrm{~mm} \times 22.0 \mathrm{~mm}$. A projectile is dragged by an external force horizontally towards the chamber center and upwards. The positions of the projectile are shown with white circles that are spaced $10 \mathrm{~ms}$ apart in time. In the background, a single simulation frame is shown, with the corresponding projectile position as filled circle. The colors of the background particles indicate the vertical velocity of the microparticles and are scaled between $-3 \mathrm{~mm} / \mathrm{s}$ (cyan/light gray) to $3 \mathrm{~mm} / \mathrm{s}$ (blue/dark gray). The Mach cone is clearly visible in the movement of the particles. The image was mirrored to the left to easy comparison with Fig. 13 .

yet - it might be, for instance, a laser-induced rocket force [105].

Figure 13 from 103 shows an example of a Mach cone in the PK-3 Plus laboratory under microgravity conditions. The background cloud is composed of microparticles of $2.55 \mu \mathrm{m}$ diameter. The gas pressure is $10 \mathrm{~Pa}$. The projectile moves from left to right, is decelerated in the void and then moves upwards through the microparticle cloud [106].

Figure 14 shows a frame and the projectile trajectory from the simulation corresponding to the experiment that we have just described. The charge of the background particles was set to $-2400 e$, as determined in [103]. The projectile is five times larger than the background particles and has a charge 25 times as large. A horizontal force of $1.4 \times 10^{-12} \mathrm{~N}$ drives the projectile through the microparticle cloud. A small vertical forces of $5 \times 10^{-14} \mathrm{~N}$ is applied in upwards direction. The white circles in Figure 14 mark the position of the projectile every $10 \mathrm{~ms}$. The distance between the circles increases until the projectile reaches the background cloud - it accelerates in this region. Inside the cloud, the projectile decelerates, as the decreasing distance between the white circles shows. This is equivalent to the experimental situation, where the projectile decelerated inside the microparticle cloud from $8 \mathrm{~cm} / \mathrm{s}$ to $3.7 \mathrm{~cm} / \mathrm{s}$ [103]. The reason for the deceleration is the interaction with the background particles - the projectile has to move them out of the way to be able to move.

As in the experiment, the projectile excites a Mach cone in the back- 
ground cloud. This is visible in the colors of the microparticles (see Fig. 14). The microparticles in front of the projectile move away from the projectile; the particles in the region above the projectile move upwards, those in the region below it move downwards. In the region behind the projectile, the particles move to fill the void left by the projectile. Thus, the particles in the region above the projectile path move downwards and the particles in the lower region move upwards.

The angle of the Mach cone increases in the simulation as in the experiment while the projectile moves through the cloud. This is due to the fact that the projectile is decelerating while moving on its trajectory inside the cloud.

The projectile moves upwards and finally penetrates the cloud above the void, where it is again decelerated by the smaller microparticles. In the experiment, the projectile reaches the region above the chamber center and is consequently accelerated into the upper cloud, but its trajectory forms a steeper angle inside the void than in the simulation.

The similarities between the simulation and the experiment break down when the projectile reaches the midplane of the chamber: In the simulation, we have applied reflecting boundary conditions at the vertical midplane of the chamber. In the experiment, the projectile moves into the right half of the chamber, in our simulation, it is reflected by the boundary and starts moving backwards/to the left. This means that it moves in opposite direction to the driving force, which then in turn decelerates it and turns it around again. In the experiment, the projectile continues a straight course through the upper particle cloud until it reaches the cloud edge (see Fig. 13).

\section{Concluding remarks}

In this paper, we have presented a new simulation of complex plasmas. It consists of a coupled fluid simulation of the capacitively coupled plasma chamber and a molecular dynamics simulation of the microparticles. At the present stage, both parts of the simulation are two-dimensional. Nevertheless, we were able to qualitatively reproduce many phenomena of experiments with complex plasmas. For instance, the ambipolar electric field automatically confines particles placed at the edge of the chamber. The particles then move towards the center, where they form a cloud with a central, particle free void. The particles in our simulation arrange by size, with smaller particles closer to the center than larger ones. We also observe lane formation when small particles penetrate a cloud of larger ones, and Mach cones when very fast projectiles penetrate the particle cloud.

In the future, we plan to complete the coupling between the two simulations and include the effects of the microparticles on the plasma. We also plan to extend the simulations to three dimensions. 


\section{Acknowledgments}

We would like to thank Emi Kawamura and Michael Lieberman for providing the original plasma model and for offering help and advise in applying the model. We also thank Jhih-Wei Chu, Alexei Ivlev, Sergey Khrapak, Hubertus Thomas and Sergey Zhdanov for helpful discussions and support.

This research was supported by a Marie Curie International Outgoing Fellowship within the 7th European Community Framework Program. The authors acknowledge partial support from the Department of Energy, Office of Fusion Science Plasma Science Center. The PK-3 Plus project is funded by the space agency of the Deutsches Zentrum für Luft- und Raumfahrt with funds from the German Federal Ministry for Economy and Technology according to a resolution of the Deutscher Bundestag under grant numbers 50 WP 0203 and 50 WM 1203.

\section{References}

[1] H. Thomas, G. E. Morfill, V. Demmel, J. Goree, B. Feuerbacher, and D. Möhlmann, Phys. Rev. Lett. 73, 652 (1994).

[2] J. H. Chu and L. I, Phys. Rev. Lett. 72, 4009 (1994).

[3] Y. Hayashi and K. Tachibana, Jpn. J. Appl. Phys. 33, L804 (1994).

[4] G. E. Morfill and A. V. Ivlev, Rev. Mod. Phys. 81, 1353 (2009).

[5] F. Melandsø and J. Goree, Phys. Rev. E 52, 5312 (1995).

[6] M. R. Akdim and W. J. Goedheer, Phys. Rev. E 65, 015401(R) (2001).

[7] M. R. Akdim and W. J. Goedheer, Phys. Rev. E 67, 066407 (2003).

[8] M. R. Akdim and W. J. Goedheer, J. Appl. Phys. 94, 104 (2003).

[9] G. Gozadinos, A. V. Ivlev, and J. P. Boeuf, New J. Phys. 5, 32 (2003).

[10] V. Land, L. S. Matthews, T. W. Hyde, and D. Bolser, Phys. Rev. E 81, 056402 (2010).

[11] M. R. Akdim and W. J. Goedheer, Phys. Rev. E 67, 056405 (2003).

[12] V. Land and W. J. Goedheer, New J Phys 8, 8 (2006).

[13] D. Block, J. Carstensen, P. Ludwig, W. Miloch, F. Greiner, A. Piel, M. Bonitz, and A. Melzer, Contrib. Plasma Phys. (2012).

[14] S. J. Choi and M. J. Kushner, J. Appl. Phys. 75, 3351 (1994).

[15] G. Lapenta, Phys. Rev. Lett. 75, 4409 (1995). 
[16] V. A. Schweigert, I. V. Schweigert, A. Melzer, A. Homann, and A. Piel, Phys. Rev. E 54, 4155 (1996).

[17] G. Lapenta, Phys. Plasmas 6, 1442 (1999).

[18] D. Winske, M. S. Murillo, and M. Rosenberg, Phys. Rev. E 59, 2263 (1999).

[19] J. W. Manweiler, T. P. Armstrong, and T. E. Cravens, J. Plasmas Phys. 63, 269 (2000).

[20] D. Winske, W. Daughton, D. S. Lemons, and M. S. Murillo, Phys. Plasmas 7, 2320 (2000).

[21] I. H. Hutchinson, Plasma Phys. Control. Fusion 44, 1953 (2002).

[22] I. H. Hutchinson, Plasma Phys. Control. Fusion 45, 1477 (2003).

[23] I. H. Hutchinson, Plasma Phys. Control. Fusion 47, 71 (2005).

[24] I. H. Hutchinson, Plasma Phys. Control. Fusion 48, 185 (2006).

[25] K. Matyash and R. Schneider, J. Plasma Phys. 72, 809 (2006).

[26] R. Smirnov, Y. Tomita, D. Tskhakaya, and T. Takizuka, Contrib. Plasma Phys. 46, 623 (2006).

[27] K. Matyash, R. Schneider, F. Taccogna, A. Hatayama, S. Longo, M. Capitelli, D. Tskhakaya, and F. X. Bronold, Contrib. Plasma Phys. 47, 595634 (2007).

[28] W. J. Miloch, H. L. Pécseli, and J. Trulsen, Nonlin. Processes Geophys. 14, 575 (2007).

[29] W. J. Miloch, J. Trulsen, and H. L. Pécseli, Phys. Rev. E 77, 056408 (2008).

[30] W. J. Miloch, S. V. Vladimirov, L. Pcseli, and J. Trulsen, Phys. Plasmas 16, 023703 (2009).

[31] W. J. Miloch, S. V. Vladimirov, H. L. Pécseli, and J. Trulsen, New J. Phys. 11, 043005 (2009).

[32] I. H. Hutchinson, Phys Plasmas 18, 032111 (2011).

[33] W. J. Miloch, S. V. Vladimirov, and V. V. Yaroshenko, EPL 101, 15001 (2013).

[34] A. P. Sun, M. Geng, and X. M. Qiu, J. Plasma Phys. 64, 263 (2000). 
[35] S. A. Maiorov, S. V. Vladimirov, and N. F. Cramer, Phys. Rev. E 63, 017401 (2000).

[36] M. A. Olevanov, Y. A. Mankelevich, and T. V. Rakhimova, JETP 96, 444 (2003).

[37] S. V. Vladimirov, S. A. Maiorov, and N. F. Cramer, Phys. Rev. E 67, 016407 (2003).

[38] J. P. Boeuf, Phys. Rev. A 46, 7910 (1992).

[39] Y. Chutov and W. J. Goedheer, IEEE Trans. Plas. Sci. 31, 606 (2003).

[40] P. Belenguer, J. P. Blondeau, L. Boufendi, M. Toogood, A. Plain, A. Bouchoule, C. Laure, and J. P. Boeuf, Phys. Rev. A 46, 7923 (1992).

[41] G. Lapenta and J. U. Brackbill, Plasma Sourc. Sci. Techn. 6, 61 (1997).

[42] I. V. Schweigert, A. L. Alexandrov, D. A. Ariskin, F. M. Peeters, I. Stefanović, E. Kovačević, J. Berndt, and J. Winter, Phys. Rev. E 78, 026410 (2008).

[43] Y. K. Khodataev, S. A. Khrapak, A. P. Nefedov, and O. F. Petrov, Phys. Rev. E 57, 7086 (1998).

[44] D. Samsonov, J. Goree, Z. W. Ma, A. Bhattacharjee, H. M. Thomas, and G. E. Morfill, Phys. Rev. Lett. 83, 3649 (1999).

[45] H. Ohta and S. Hamaguchi, Phys. Plasmas 7, 4506 (2000).

[46] R. A. Quinn and J. Goree, Phys. Rev. E 61, 3033 (2000).

[47] O. S. Vaulina, A. P. Nefedov, O. F. Petrov, and V. E. Fortov, J. Exp. Theo. Phys. 91, 1147 (2000).

[48] O. S. Vaulina and S. A. Khrapak, JETP 92, 228 (2001).

[49] W. J. Goedheer and M. R. Akdim, Phys. Rev. E 68, 045401(R) (2003).

[50] K. Jiang, L.-J. Hou, Y.-N. Wang, and Z. L. Mišković, Phys. Rev. E 73, 016404 (2006).

[51] B. A. Klumov, M. Rubin-Zuzic, and G. E. Morfill, JETP Lett. 84, $542(2006)$.

[52] Y. H. Liu, Z. Y. Chen, M. Y. Yu, and A. Bogaerts, Phys. Rev. E 74, 056401 (2006).

[53] Y. Liu and L. Y. Chew, J. Phys. A: Math. Theor. 40, 10383 (2007). 
[54] D. Block, S. Käding, A. Melzer, A. Piel, H. Baumgartner, and M. Bonitz, Phys. Plasmas 15, 040701 (2008).

[55] H. Kählert, P. Ludwig, H. Baumgartner, M. Bonitz, D. Block, S. Käding, A. Melzer, and A. Piel, Phys. Rev. E 78, 036408 (2008).

[56] T. Ott, M. Bonitz, Z. Donkó, and P. Hartmann, Phys. Rev. E 78, 026409 (2008).

[57] K. Jiang, C.-R. Du, K. R. Sütterlin, A. V. Ivlev, and G. E. Morfill, Europhys. Lett. 92, 65002 (2010).

[58] A. Wysocki, C. Räth, A. V. Ivlev, K. R. Sütterlin, H. M. Thomas, S. Khrapak, S. Zhdanov, V. E. Fortov, A. M. Lipaev, V. I. Molotkov, et al., Phys. Rev. Lett. 105, 045001 (2010).

[59] L. Couëdel, S. K. Zhdanov, A. V. Ivlev, V. Nosenko, H. M. Thomas, and G. E. Morfill, Phys. Plasmas 18, 083707 (2011).

[60] K. Jiang, L.-J. Hou, A. V. Ivlev, Y.-F. Li, C.-R. Du, H. M. Thomas, G. E. Morfill, and K. R. Sütterlin, Europhys. Lett. 93, 55001 (2011).

[61] T. Reichstein and A. Piel, Phys. Plasmas 18, 083705 (2011).

[62] V. A. Schweigert, I. V. Schweigert, V. Nosenko, and J. Goree, Phys. Plasmas 9, 4465 (2002).

[63] K. Jiang, Y. fang Li, T. Shimizu, U. Konopka, H. M. Thomas, and G. E. Morfill, Phys. Plasmas 16, 123702 (2009).

[64] O. S. Vaulina, A. P. Nefedov, O. F. Petrov, and S. A. Khrapak, JETP 88, 1130 (1999).

[65] I. V. Schweigert, V. A. Schweigert, V. M. Bedanov, A. Melzer, A. Homann, and A. Piel, JETP 87, 905 (1998).

[66] K. Matyash, R. Schneider, R. Ikkurthi, L. Lewerentz, and A. Melzer, Plasma Phys. Control. Fusion 52, 124016 (2010).

[67] P. Agarwal and S. L. Girshick, Plasma Sourc. Sci. Techn. 21, 055023 (2012).

[68] F. Melands $\varnothing$ and J. Goree, J. Vac. Sci. Technol. A 14, 511 (1996).

[69] F. Melandsø, Phys. Rev. E 55, 7495 (1997).

[70] Z. Yu, L. Jinyuan, L. Yue, and W. Xiaogang, Plasma Sci. Tech. 8, 176 (2006). 
[71] M. Romé, F. Cavaliere, M. Cavenago, M. Ikram, F. Lepreti, G. Maero, B. Paroli, and R. Pozzoli, in AIP Conf. Proc. (2012).

[72] S. J. Choi, P. L. G. Ventzek, R. J. Hoekstra, and M. J. Kushner, Plasma Sources Sci. Tech. 3, 418 (1994).

[73] H. H. Hwang and M. J. Kushner, J. Appl. Phys. 82, 2106 (1997).

[74] H. H. Hwang, E. R. Keiter, and M. J. Kushner, J. Vac. Sci. Technol. A 16, 2454 (1998).

[75] V. Vyas, G. A. Hebner, and M. J. Kushner, J. Appl. Phys 92, 6451 (2002).

[76] V. Vyas and M. J. Kushner, J. Appl. Phys. 97, 043303 (2005).

[77] E. Kawamura, D. B. Graves, and M. A. Lieberman, Plasma Sourc. Sci. Techn. 20, 035009 (2011).

[78] S. A. Khrapak, B. A. Klumov, P. Huber, V. I. Molotkov, A. M. Lipaev, V. N. Naumkin, A. V. Ivlev, H. M. Thomas, M. Schwabe, G. E. Morfill, et al., Phys. Rev. E 85, 066407 (2012).

[79] O. Havnes, G. E. Morfill, and C. K. Goertz, J. Geophys. Res. 89, 10999 (1984).

[80] C.-C. Hsu, M. A. Nierode, J. W. Coburn, and D. B. Graves, J. Phys. D: Appl. Phys. 39, 3272 (2006).

[81] I. Lee, D. B. Graves, and M. A. Lie, Plasma Sourc. Sci. Techn. 17, 05018 (2008).

[82] H. M. Thomas, G. E. Morfill, V. E. Fortov, A. V. Ivlev, V. I. Molotkov, A. M. Lipaev, T. Hagl, H. Rothermel, S. A. Khrapak, R. K. Sütterlin, et al., New J. Phys. 10, 033036 (2008).

[83] S. Plimpton, J. Comp. Phys. 117, 1 (1995), URL http://lammps. sandia.gov.

[84] T. Matsoukas and M. Russel, J. Appl. Phys. 77, 4285 (1995).

[85] P. Epstein, Phys. Rev. 23, 710 (1924).

[86] U. Konopka, Ph.D. thesis, Ruhr-Universität-Bochum (2000).

[87] Lammps documentation, online (2013), URL http://lammps . sandia. gov/doc/

[88] W. Goedheer, V. Land, and J. Venema, Contrib. Plasma Phys. 49, 199 (2009). 
[89] S. A. Khrapak, A. V. Ivlev, G. E. Morfill, and H. M. Thomas, Phys. Rev. E 66, 046414 (2002).

[90] A. V. Ivlev, S. K. Zhdanov, S. A. Khrapak, and G. E. Morfill, Plasma Phys. Control. Fusion 46, B267 (2004).

[91] I. H. Hutchinson, in New Vistas in Dusty Plasmas (2006).

[92] S. A. Khrapak, Phys. Plasmas 20, 013703 (2013).

[93] M. Bonitz, P. Ludwig, H. Baumgartner, C. Henning, A. Filinov, D. Block, O. Arp, A. Piel, S. Käding, Y. Ivanov, et al., Phys. Plasmas 15, 055704 (2008).

[94] A. P. Nefedov, G. E. Morfill, V. E. Fortov, H. M. Thomas, H. Rothermel, T. Hagl, A. V. Ivlev, M. Zuzic, B. A. Klumov, A. M. lppaev, et al., New Journ. Phys. 5, 33 (2003).

[95] V. Land, E. Shen, B. Smith, L. Matthews, and T. Hydes, New J. Phys. 11, 063024 (2009).

[96] M. Klindworth, O. Arp, and A. Piel, J. Phys. D: Appl. Phys. 39, 1095 (2006).

[97] K. R. Sütterlin, A. Wysocki, A. V. Ivlev, C. Räth, H. M. Thomas, M. Rubin-Zuzic, W. J. Goedheer, V. E. Fortov, A. M. Lipaev, V. I. Molotkov, et al., Phys. Rev. Lett. 102, 085003 (2009).

[98] D. A. Law, W. H. Steel, B. M. Annaratone, and J. E. Allen, Phys. Rev. Lett. 80, 4189 (1998).

[99] G. E. Morfill, H. M. Thomas, U. Konopka, H. Rothermel, M. Zuzic, A. Ivlev, and J. Goree, Phys. Rev. Lett. 83, 1598 (1999).

[100] V. I. Vladimirov, L. V. Deputatova, A. P. Nefedov, V. E. Fortov, V. A. Rykov, and A. V. Khudyakov, J. Exp. Theo. Phys. 93, 313 (2001).

[101] G. Uchida, S. Iizuka, T. Kamimura, and N. Sato, Phys. Plasmas 16, 053707 (2009).

[102] C.-R. Du, K. R. Sütterlin, K. Jiang, C. Räth, A. V. Ivlev, S. Khrapak, M. Schwabe, H. M. Thomas, V. E. Fortov, A. M. Lipaev, et al., New J. Phys. 14, 073058 (2012).

[103] M. Schwabe, K. Jiang, S. Zhdanov, T. Hagl, P. Huber, A. V. Ivlev, A. M. Lipaev, V. N. Naumkin, K. R. Sütterlin, H. M. Thomas, et al., EPL 96, 55001 (2011). 
[104] K. Jiang, V. Nosenko, Y. F. Li, M. Schwabe, U. Konopka, A. V. Ivlev, V. E. Fortov, V. I. Molotkov, A. M. Lipaev, O. F. Petrov, et al., EPL 85, 45002 (2009).

[105] V. Nosenko, A. V. Ivlev, and G. E. Morfill, Phys. Plasmas 17, 123705 (2010).

[106] D. I. Zhukhovitskii, V. E. Fortov, V. I. Molotkov, A. M. Lipaev, V. N. Naumkin, H. M. Thomas, A. V. Ivlev, M. Schwabe, and G. E. Morfill, Phys. Rev. E 86, 016401 (2012). 